\title{
APPLICATION OF DIFFERENTIAL TRANSFORM METHOD TO SOLVE LINEAR,NON-LINEAR REACTION CONVECTION DIFFUSION AND CONVECTION DIFFUSION PROBLEM
}

\author{
Yogeshwari Patel $^{1 \S}$, Jayesh M. Dhodiya ${ }^{2}$ \\ ${ }^{1,2}$ Applied Mathematics \& Humanities Department \\ Ichchhanath, SVNIT, Surat Gujarat 395007, INDIA
}

\begin{abstract}
The aim of this paper is to present the semi-analytical method known as differential transform method (DTM) for solving various linear and non linear convection diffusion and reaction convection problems, arising in physics, where particles, energy, or other physical quantities are transferred inside a physical system due to two process: Diffusion and convection. To illustrate the capability and reliability of the method, some cases have been discussed. The solution has been obtaining in form of series solution which can be easily converted into the exact solution.
\end{abstract}

AMS Subject Classification: 35C05, 35E $15,35 \mathrm{~F} 10$

Key Words: linear convection diffusion equation, non-linear convection diffusion equation, differential transform method

\section{Introduction}

Convection-Diffusion problems arises very frequently in applied sciences and engineering as it describes the physical phenomena where particles, energy, or the other physical quantities are transferred inside a physical system, migration of contaminations in a stream smoke plume in atmosphere, dispersion of chemical in reactors, tracers dispersion in porous medium etc.. Mathematical formulation of such kind of physical phenomena is represented by convection-diffusion

\footnotetext{
Received: $\quad$ May 8, 2016

Revised: June 9, 2016

Published: September 30, 2016

$\S_{\text {Correspondence author }}$
}

(c) 2016 Academic Publications, Ltd. url: www.acadpubl.eu 
equation due to the combine effect of two processes: diffusion and convection. General form of the convection diffusion equation is given by:

$$
\frac{\partial u}{\partial t}=\nabla \cdot(D \cdot \nabla u)-\nabla \cdot(\vec{v} u)+R .
$$

Here $\mathrm{u}$ is the variable of interest, $\mathrm{D}$ is the diffusivity, is the average velocity that the quantity is moving, $\mathrm{R}$ describes the source or sinks of the quantity. In the literature many methods such as vibrational iterative method [6], Adomain Decomposition Method[4], Variational Homotopy Perturbation Method[7],combining Laplace Transform method[5] has been applied to solve reaction diffusion convection problem as well as convection diffusion problems. The main aim this paper is to apply Differential Transform Method to reaction diffusion or convection diffusion problems to overcome the disadvantages of the existing methods available in the literature. Using DTM we get reduce the computation work as well as it convergence to the exact solution rapidly. Section 2 describes the basic idea of two dimensional transform method. Section 3 describe the solution of various linear and non-linear convection diffusion equation arising in physical phenomena using differential transform method.

\section{Two Dimensional Differential Transform}

In order to solve the PDE by two dimensional transform its basic theory is stated in this section.

Definition 1. Two-Dimensional Differential transform of the function $\mathrm{w}(\mathrm{x}, \mathrm{y})$ is defined as follows (see [1], [2], [3])

$$
W(k, h)=\frac{1}{k ! h !}\left[\frac{\partial^{k+h}}{\partial x^{k} \partial y^{h}} w(x, y)\right] .
$$

In (1), w(x,y) is the original function and $W(k, h)$ is the transformed $f$ which is called $T$ function.

Definition 2. Differential inverse transform of $W(k, h)$ is defined as follows

$$
w(x, y)=\sum_{k=0}^{\infty} \sum_{h=0}^{\infty} W(k, h) x^{k} y^{h} .
$$

Using (1) and (2), we obtain

$$
W(k, h)=\sum_{k=0}^{\infty} \sum_{h=0}^{\infty} \frac{1}{k ! h !}\left[\frac{\partial^{k+h}}{\partial x^{k} \partial y^{h}} w(x, y)\right] x^{k} y^{h} .
$$




\begin{tabular}{|l|l|}
\hline Original Function & Transform Function \\
\hline$u(x, y) \pm v(x, y)$ & $U(k, h) \pm V(k, h)$ \\
\hline$\alpha v(x, y)$ & $\lambda U(k, h)$ \\
\hline$\frac{\partial u(x, y)}{\partial x}$ & $(k+1) U(k+1, h)$ \\
\hline$\frac{\partial u(x, y)}{\partial y}$ & $(h+1) U(k, h+1)$ \\
\hline$\frac{\partial^{r+s} u(x, y)}{\partial x^{r} \partial y^{s}}$ & $(k+1)(k+2) \ldots(k+r)(h+1)(h+2) \ldots(h+s) U(k+r, h+s)$ \\
\hline$v(x, y) w(x, y)$ & $\sum_{r=0}^{k} \sum_{s=0}^{h} V(r, h-s) W(k-r, s)$ \\
\hline$x^{m} y^{n}$ & $U(k, h)=\delta(k-m, h-n)= \begin{cases}1 & k=\text { m and } h=n \\
0 & \text { otherwise }\end{cases}$ \\
\hline$e^{\lambda(x+y)}$ & $\frac{\lambda^{(k+h)}}{k ! h !}$ \\
\hline
\end{tabular}

Table 1: Fundamental operations of two-dimensional DTM

\section{Numerical Examples}

In this section, we apply Differential Transform form method to the convection diffusion as well as reaction convection diffusion problems. Example 1 and 2 are convection diffusion problems and Example 3 and 4 are reaction diffusion problems.

Example 1. Consider the following diffusion convection problem[7]

$$
\frac{\partial u}{\partial t}=\frac{\partial^{2} u}{\partial x^{2}}-u
$$

with the initial condition $u(x, 0)=x+e^{-x}$.

Applying the differential transform to (5) we get

$$
(h+1) U(k, h+1)=(k+2)(k+1) U(k+2, h)-U(k, h) .
$$

Applying DTM to (6) we receive

$$
U(0,0)=1, \quad U(1,0)=0, \quad U(k, 0)=\frac{(-1)^{k}}{k !}, \quad k \geq 2 .
$$

Using (7) and (8) we obtain the coefficient, see next table.

Using(3) we get

$$
u(x, t)=\sum_{k=0}^{\infty} \sum_{h=0}^{\infty} U(k, h) x^{k} t^{h}
$$




\begin{tabular}{|c|c|c|c|c|c|c|c|c|c|}
\hline $\mathrm{k}$ & $\mathrm{h}=0$ & $\mathrm{~h}=1$ & $\mathrm{~h}=2$ & $\mathrm{~h}=3$ & $\mathrm{~h}=4$ & $\mathrm{~h}=5$ & $\mathrm{~h}=6$ & $\mathrm{~h}=7$ & $\mathrm{~h}=8$ \\
\hline 0 & 1 & 0 & 0 & 0 & 0 & 0 & 0 & 0 & 0 \\
\hline 1 & 0 & $-\frac{1}{1 ! 1 !}$ & $\frac{1}{1 ! 2 !}$ & $-\frac{1}{1 ! 3 !}$ & $\frac{1}{1 ! 4 !}$ & $-\frac{1}{1 ! 5 !}$ & $\frac{1}{1 ! 6 !}$ & $-\frac{1}{1 ! 7 !}$ & $\frac{1}{1 ! 8 !}$ \\
\hline 2 & $\frac{1}{2 !}$ & 0 & 0 & 0 & 0 & 0 & 0 & 0 & 0 \\
\hline 3 & $\frac{-1}{3 !}$ & 0 & 0 & 0 & 0 & 0 & 0 & 0 & 0 \\
\hline 4 & $\frac{1}{4 !}$ & 0 & 0 & 0 & 0 & 0 & 0 & 0 & 0 \\
\hline 5 & $\frac{-1}{5 !}$ & 0 & 0 & 0 & 0 & 0 & 0 & 0 & 0 \\
\hline 6 & $\frac{1}{6 !}$ & 0 & 0 & 0 & 0 & 0 & 0 & 0 & 0 \\
\hline 7 & $-\frac{1}{7 !}$ & 0 & 0 & 0 & 0 & 0 & 0 & 0 & 0 \\
\hline 8 & $\frac{1}{8 !}$ & 0 & 0 & 0 & 0 & 0 & 0 & 0 & 0 \\
\hline 9 & $-\frac{1}{9 !}$ & 0 & 0 & 0 & 0 & 0 & 0 & 0 & 0 \\
\hline 10 & $\frac{1}{10 !}$ & 0 & 0 & 0 & 0 & 0 & 0 & 0 & 0 \\
\hline
\end{tabular}

Table 2: Coefficient Table of $\mathrm{U}(\mathrm{k}, \mathrm{h})$

Using Table 1 we get following series solution

$$
\begin{aligned}
& u(x, t)=\left[1+\frac{x^{2}}{2 !}-\frac{x^{3}}{3 !}+\frac{x^{4}}{4 !}-\frac{x^{5}}{5 !}+\frac{x^{6}}{6 !}-\frac{x^{7}}{7 !}+\frac{x^{8}}{8 !}-\frac{x^{9}}{9 !}+\frac{x^{10}}{10 !}+\cdots\right] \\
& +x-x-\frac{x t}{1 !}+\frac{x t^{2}}{1 ! 2 !}-\frac{x t^{3}}{1 ! 3 !}+\frac{x t^{4}}{1 ! 4 !}-\frac{x t^{5}}{1 ! 5 !}+\frac{x t^{6}}{1 ! 6 !}-\frac{x t^{7}}{1 ! 7 !}+\frac{x t^{8}}{1 ! 8 !} \cdots
\end{aligned}
$$

Equation (10) can be expressed in the closed form (exact solution) as follows

$$
u(x, t)=e^{-x}+x e^{-t} .
$$

Example 2. Consider the following diffusion convection problem[5]

$$
\frac{\partial u}{\partial t}=\frac{\partial^{2} u}{\partial x^{2}}-\frac{u}{4}, \quad x, t \in R,
$$

with initial condition $u(x, 0)=\frac{x}{2}+e^{-\frac{x}{2}}$.

Applying the differential transform to (12) we receive

$$
(h+1) U(k, h+1)=(k+2)(k+1) U(k+2, h)-\frac{1}{4} U(k, h) .
$$

Applying DTM to (13) we have

$$
U(0,0)=1, \quad U(1,0)=0, \quad U(k, 0)=\frac{(-1)^{k}}{2^{2} k !}, \quad k \geq 2 .
$$

Using (14) and (15) we obtain the coefficient listed in the next table. 

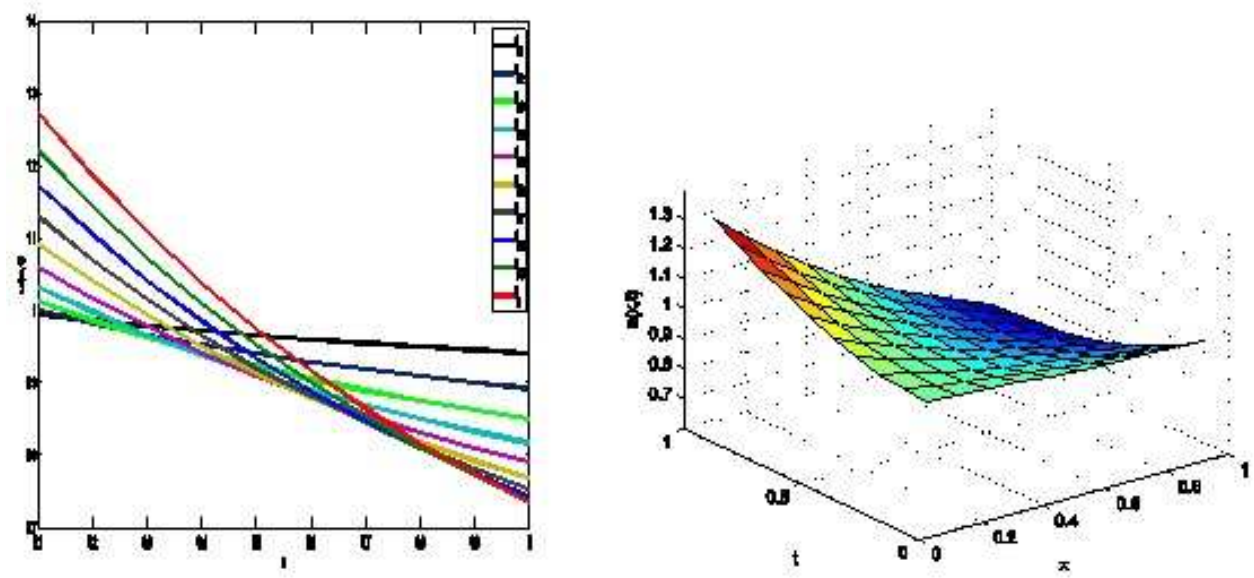

Figure 1: Graphical representation of $u(x, t)$ w.r.t time and distance

\begin{tabular}{|c|c|c|c|c|c|c|c|c|c|}
\hline & 0 & 1 & 2 & 3 & 4 & 5 & 6 & 7 & 8 \\
\hline 0 & 1 & 0 & 0 & 0 & 0 & 0 & 0 & 0 & 0 \\
\hline 1 & 0 & $-\frac{1}{2^{3} 1 !}$ & $\frac{1}{2^{5} 2 !}$ & $-\frac{1}{2^{7} 3 !}$ & $\frac{1}{2^{9} 4 !}$ & $-\frac{1}{2^{11} 5 !}$ & $\frac{1}{2^{13} 6 !}$ & $-\frac{1}{2^{15} 7 !}$ & $\frac{1}{2^{17} 8 !}$ \\
\hline 2 & $\frac{1}{2^{2} 2 !}$ & 0 & 0 & 0 & 0 & 0 & 0 & 0 & 0 \\
\hline 3 & $\frac{-1}{2^{3} 3 !}$ & 0 & 0 & 0 & 0 & 0 & 0 & 0 & 0 \\
\hline 4 & $\frac{1}{2^{4} 4 !}$ & 0 & 0 & 0 & 0 & 0 & 0 & 0 & 0 \\
\hline 5 & $\frac{-1}{2^{5} 5 !}$ & 0 & 0 & 0 & 0 & 0 & 0 & 0 & 0 \\
\hline 6 & $\frac{1}{2^{6} 6 !}$ & 0 & 0 & 0 & 0 & 0 & 0 & 0 & 0 \\
\hline 7 & $-\frac{1}{2^{7} 7 !}$ & 0 & 0 & 0 & 0 & 0 & 0 & 0 & 0 \\
\hline 8 & $\frac{1}{2^{8} 8 !}$ & 0 & 0 & 0 & 0 & 0 & 0 & 0 & 0 \\
\hline 9 & $-\frac{1}{2^{9} 9 !}$ & 0 & 0 & 0 & 0 & 0 & 0 & 0 & 0 \\
\hline 10 & $\frac{1}{2^{10} 10 !}$ & 0 & 0 & 0 & 0 & 0 & 0 & 0 & 0 \\
\hline
\end{tabular}

Table 3: Coefficient Table of $\mathrm{U}(\mathrm{k}, \mathrm{h})$

Using equation (2) and Table 2 we get following series solution:

$$
\begin{gathered}
u(x, t)=\left[1+\frac{x^{2}}{2^{2} 2 !}-\frac{x^{3}}{2^{3} 3 !}+\frac{x^{4}}{2^{4} 4 !}-\frac{x^{5}}{2^{5} 5 !}+\frac{x^{6}}{2^{6} 6 !}-\frac{x^{7}}{2^{7} 7 !}+\frac{x^{8}}{2^{8} 8 !}-\frac{x^{9}}{2^{9} 9 !}\right. \\
\left.+\frac{x^{10}}{2^{10} 10 !}+\cdots\right]-\frac{x}{2}+\frac{x}{2} \\
-\frac{x t}{2^{3} 1 !}+\frac{x t^{2}}{2^{5} 2 !}-\frac{x t^{3}}{2^{7} 3 !}+\frac{x t^{4}}{2^{9} 4 !}-\frac{x t^{5}}{2^{11} 5 !}+\frac{x t^{6}}{2^{13} 6 !}-\frac{x t^{7}}{2^{15} 7 !}+\frac{x t^{8}}{2^{17} 8 !} \cdots
\end{gathered}
$$

Which series solution of example 2 and it can be converted into closed form 

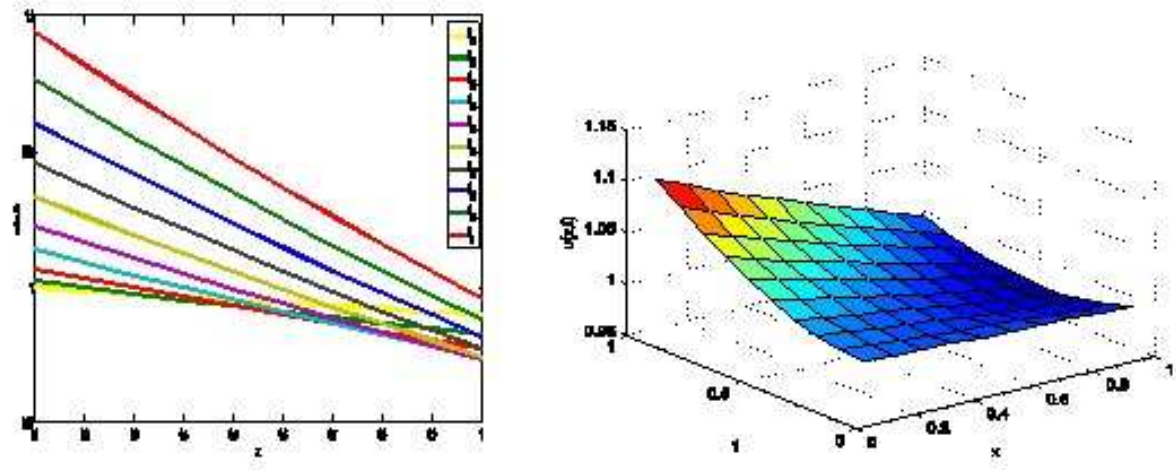

Figure 2: Graphical Representation of $u(x, t)$ w.r.t time and distance

given as follows

$$
u(x, t)=e^{-\frac{x}{2}}+\frac{x}{2} e^{-\frac{t}{4}}
$$

Example 3. Consider the reaction convection diffusion problem (nonlinear) $[6]$

$$
\frac{\partial u}{\partial t}=\frac{\partial^{2} u}{\partial x^{2}}+u \frac{\partial u}{\partial x}+u-u^{2}
$$

with initial and boundary condition $u(x, t)=1+e^{x} ; u(0, t)=1+e^{t}$.

Applying DTM on (18), (19) and (20) we have

$$
\begin{aligned}
& (h+1) U(k, h+1) \\
& =(k+2)(k+1) U(k+2, h)+\sum_{r=0}^{k} \sum_{s=0}^{h} U(r, h-s)(k-r+1) U(k-r+1) \\
& \quad+U(k, h)-\sum_{r=0}^{k} \sum_{s=0}^{h} U(r, h-s) U(k-r, s), \\
& U(0,0)=2, \quad U(k, 0)=\frac{1}{k !}, k \geq 1, \quad U(0,0)=2, \quad U(0, h)=\frac{1}{h !}, h \geq 1 .
\end{aligned}
$$

Using (21), (22), and (23) we obtain the results listed in Table 4. Using Table 3 we get following series solution

$$
u(x, t)=1+\left[1+\frac{x}{1 !}+\frac{x^{2}}{2 !}+\frac{x^{3}}{3 !}+\frac{x^{4}}{4 !}+\cdots\right]
$$




\begin{tabular}{|c|c|c|c|c|c|c|c|c|c|}
\hline & 0 & 1 & 2 & 3 & 4 & 5 & 6 & 7 & 8 \\
\hline 0 & 2 & $\frac{1}{0 ! 1 !}$ & $\frac{1}{0 ! 2 !}$ & $\frac{1}{0 ! 3 !}$ & $\frac{1}{0 ! 4 !}$ & $\frac{1}{0 ! 5 !}$ & $\frac{1}{0 ! 6 !}$ & $\frac{1}{0 ! 7 !}$ & $\frac{1}{0 ! 8 !}$ \\
\hline 1 & $\frac{1}{0 ! 1 !}$ & $\frac{1}{1 ! 1 !}$ & $\frac{1}{1 ! 2 !}$ & $\frac{1}{1 ! 3 !}$ & $\frac{1}{1 ! 4 !}$ & $\frac{1}{1 ! 5 !}$ & $\frac{1}{1 ! 6 !}$ & $\frac{1}{1 ! 7 !}$ & $\frac{1}{1 ! 8 !}$ \\
\hline 2 & $\frac{1}{0 ! 2 !}$ & $\frac{1}{2 ! 1 !}$ & $\frac{1}{2 ! 2 !}$ & $\frac{1}{2 ! 3 !}$ & $\frac{1}{2 ! 4 !}$ & $\frac{1}{2 ! 5 !}$ & $\frac{1}{2 ! 6 !}$ & $\frac{1}{2 ! 7 !}$ & $\frac{1}{2 ! 8 !}$ \\
\hline 3 & $\frac{1}{0 ! 3 !}$ & $\frac{1}{3 ! 1 !}$ & $\frac{1}{3 ! 2 !}$ & $\frac{1}{3 ! 3 !}$ & $\frac{1}{3 ! 4 !}$ & $\frac{1}{3 ! 5 !}$ & $\frac{1}{3 ! 6 !}$ & $\frac{1}{3 ! 7 !}$ & $\frac{1}{3 ! 8 !}$ \\
\hline 4 & $\frac{1}{0 ! 4 !}$ & $\frac{1}{4 ! 1 !}$ & $\frac{1}{4 ! 2 !}$ & $\frac{1}{4 ! 3 !}$ & $\frac{1}{4 ! 4 !}$ & $\frac{1}{4 ! 5 !}$ & $\frac{1}{4 ! 6 !}$ & $\frac{1}{4 ! 7 !}$ & $\frac{1}{4 ! 8 !}$ \\
\hline 5 & $\frac{1}{0 ! 5 !}$ & $\frac{1}{5 ! 1 !}$ & $\frac{1}{5 ! 2 !}$ & $\frac{1}{5 ! 3 !}$ & $\frac{1}{5 ! 4 !}$ & $\frac{1}{5 ! 5 !}$ & $\frac{1}{5 ! 6 !}$ & $\frac{1}{5 ! 7 !}$ & $\frac{1}{5 ! 8 !}$ \\
\hline 6 & $\frac{1}{0 ! 6 !}$ & $\frac{1}{6 ! 1 !}$ & $\frac{1}{6 ! 2 !}$ & $\frac{1}{6 ! 3 !}$ & $\frac{1}{6 ! 4 !}$ & $\frac{1}{6 ! 5 !}$ & $\frac{1}{6 ! 6 !}$ & $\frac{1}{6 ! 7 !}$ & $\frac{1}{6 ! 8 !}$ \\
\hline 7 & $\frac{1}{0 ! 7 !}$ & $\frac{1}{7 ! 1 !}$ & $\frac{1}{7 ! 2 !}$ & $\frac{1}{7 ! 3 !}$ & $\frac{1}{7 ! 4 !}$ & $\frac{1}{7 ! 5 !}$ & $\frac{1}{7 ! 6 !}$ & $\frac{1}{7 ! 7 !}$ & $\frac{1}{7 ! 8 !}$ \\
\hline 8 & $\frac{1}{0 ! 8 !}$ & $\frac{1}{8 ! 1 !}$ & $\frac{1}{8 ! 2 !}$ & $\frac{1}{8 ! 3 !}$ & $\frac{1}{8 ! 4 !}$ & $\frac{1}{8 ! 5 !}$ & $\frac{1}{8 ! 6 !}$ & $\frac{1}{8 ! 7 !}$ & $\frac{1}{8 ! 8 !}$ \\
\hline 9 & $\frac{1}{0 ! 9 !}$ & $\frac{1}{9 ! 1 !}$ & $\frac{1}{9 ! 2 !}$ & $\frac{1}{9 ! 3 !}$ & $\frac{1}{9 ! 4 !}$ & $\frac{1}{9 ! 5 !}$ & $\frac{1}{9 ! 6 !}$ & $\frac{1}{9 ! 7 !}$ & $\frac{1}{9 ! 8 !}$ \\
\hline 10 & $\frac{1}{0 ! 10 !}$ & $\frac{1}{10 ! 1 !}$ & $\frac{1}{10 ! 2 !}$ & $\frac{1}{10 ! 3 !}$ & $\frac{1}{10 ! 4 !}$ & $\frac{1}{10 ! 5 !}$ & $\frac{1}{10 ! 6 !}$ & $\frac{1}{10 ! 7 !}$ & $\frac{1}{10 ! 8 !}$ \\
\hline
\end{tabular}

Table 4: Coefficient of $U(k, h)$

$$
\begin{gathered}
+\left[1+\frac{x}{1 ! 1 !}+\frac{x^{2}}{1 ! 2 !}+\frac{x^{3}}{1 ! 3 !}+\frac{x^{4}}{1 ! 4 !}+\cdots\right] t \\
+\left[1+\frac{x}{2 ! 1 !}+\frac{x^{2}}{2 ! 2 !}+\frac{x^{3}}{2 ! 3 !}+\frac{x^{4}}{2 ! 4 !}+\cdots\right] t^{2}+\left[1+\frac{x}{3 ! 1 !}+\frac{x^{2}}{3 ! 2 !}+\frac{x^{3}}{3 ! 3 !}+\frac{x^{4}}{3 ! 4 !}+\cdots\right] t^{3} \\
+\left[1+\frac{x}{4 ! 1 !}+\frac{x^{2}}{4 ! 2 !}+\frac{x^{3}}{4 ! 3 !}+\frac{x^{4}}{4 ! 4 !}+\cdots\right] t^{4}+\cdots
\end{gathered}
$$

The series solution of Example 3 and it exact solution are

$$
u(x, t)=1+e^{x+t} .
$$

Example 4. Consider the PDE (see [6])

$$
\frac{\partial u}{\partial t}+u \frac{\partial u}{\partial x}-u(1-u)=0, \quad 0 \leq x \leq 1, t>0
$$

with initial condition

$$
u(x, 0)=e^{-x} .
$$

Applying DTM on (26) and (27) we get

$$
(h+1) U(k, h+1)=U(k, h)-\sum_{r=0}^{k} \sum_{s=0}^{h} U(r, h-s) U(k-r, s)
$$



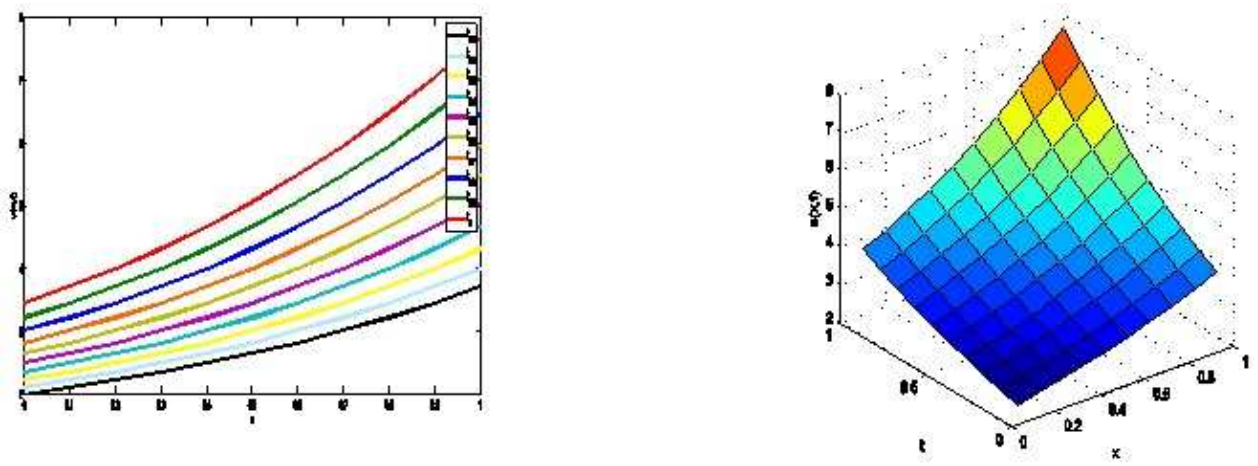

Figure 3: Graphical representation of $u(x, t)$ w.r.t time and distance

\begin{tabular}{|c|c|c|c|c|c|c|c|c|c|}
\hline & 0 & 1 & 2 & 3 & 4 & 5 & 6 & 7 & 8 \\
\hline 0 & 1 & $\frac{1}{0 ! 1 !}$ & $\frac{1}{0 ! 2 !}$ & $\frac{1}{0 ! 3 !}$ & $\frac{1}{0 ! 4 !}$ & $\frac{1}{0 ! 5 !}$ & $\frac{1}{0 ! 6 !}$ & $\frac{1}{0 ! 7 !}$ & $\frac{1}{0 ! 8 !}$ \\
\hline 1 & $-\frac{1}{0 ! 1 !}$ & $-\frac{1}{1 ! 1 !}$ & $-\frac{1}{1 ! 2 !}$ & $-\frac{1}{1 ! 3 !}$ & $-\frac{1}{1 ! 4 !}$ & $-\frac{1}{1 ! 5 !}$ & $-\frac{1}{1 ! 6 !}$ & $-\frac{1}{1 ! 7 !}$ & $-\frac{1}{1 ! 8 !}$ \\
\hline 2 & $\frac{1}{0 ! 2 !}$ & $\frac{1}{2 ! 1 !}$ & $\frac{1}{2 ! 2 !}$ & $\frac{1}{2 ! 3 !}$ & $\frac{1}{2 ! 4 !}$ & $\frac{1}{2 ! 5 !}$ & $\frac{1}{2 ! 6 !}$ & $\frac{1}{2 ! 7 !}$ & $\frac{1}{2 ! 8 !}$ \\
\hline 3 & $-\frac{1}{0 ! 3 !}$ & $-\frac{1}{3 ! 1 !}$ & $-\frac{1}{3 ! 2 !}$ & $-\frac{1}{3 ! 3 !}$ & $-\frac{1}{3 ! 4 !}$ & $-\frac{1}{3 ! 5 !}$ & $-\frac{1}{3 ! 6 !}$ & $-\frac{1}{3 ! 7 !}$ & $-\frac{1}{3 ! 8 !}$ \\
\hline 4 & $\frac{1}{0 ! 4 !}$ & $\frac{1}{4 ! 1 !}$ & $\frac{1}{4 ! 2 !}$ & $\frac{1}{4 ! 3 !}$ & $\frac{1}{4 ! 4 !}$ & $\frac{1}{4 ! 5 !}$ & $\frac{1}{4 ! 6 !}$ & $\frac{1}{4 ! 7 !}$ & $\frac{1}{4 ! 8 !}$ \\
\hline 5 & $-\frac{1}{0 ! 5 !}$ & $-\frac{1}{5 ! 1 !}$ & $-\frac{1}{5 ! 2 !}$ & $-\frac{1}{5 ! 3 !}$ & $-\frac{1}{5 ! 4 !}$ & $-\frac{1}{5 ! 5 !}$ & $-\frac{1}{5 ! 6 !}$ & $-\frac{1}{5 ! 7 !}$ & $-\frac{1}{5 ! 8 !}$ \\
\hline 6 & $\frac{1}{0 ! 6 !}$ & $\frac{1}{6 ! 1 !}$ & $\frac{1}{6 ! 2 !}$ & $\frac{1}{6 ! 3 !}$ & $\frac{1}{6 ! 4 !}$ & $\frac{1}{6 ! 5 !}$ & $\frac{1}{6 ! 6 !}$ & $\frac{1}{6 ! 7 !}$ & $\frac{1}{6 ! 8 !}$ \\
\hline 7 & $-\frac{1}{0 ! 7 !}$ & $-\frac{1}{7 ! 1 !}$ & $-\frac{1}{7 ! 2 !}$ & $-\frac{1}{7 ! 3 !}$ & $-\frac{1}{7 ! 4 !}$ & $-\frac{1}{7 ! 5 !}$ & $-\frac{1}{7 ! 6 !}$ & $-\frac{1}{7 ! 7 !}$ & $-\frac{1}{7 ! 8 !}$ \\
\hline 8 & $\frac{1}{0 ! 8 !}$ & $\frac{1}{8 ! 1 !}$ & $\frac{1}{8 ! 2 !}$ & $\frac{1}{8 ! 3 !}$ & $\frac{1}{8 ! 4 !}$ & $\frac{1}{8 ! 5 !}$ & $\frac{1}{8 ! 6 !}$ & $\frac{1}{8 ! 7 !}$ & $\frac{1}{8 ! 8 !}$ \\
\hline 9 & $-\frac{1}{0 ! 9 !}$ & $-\frac{1}{9 ! 1 !}$ & $-\frac{1}{9 ! 2 !}$ & $-\frac{1}{9 ! 3 !}$ & $-\frac{1}{9 ! 4 !}$ & $-\frac{1}{9 ! 5 !}$ & $-\frac{1}{9 ! 6 !}$ & $-\frac{1}{9 ! 7 !}$ & $-\frac{1}{9 ! 8 !}$ \\
\hline 10 & $\frac{1}{0 ! 10 !}$ & $\frac{1}{10 ! 1 !}$ & $\frac{1}{10 ! 2 !}$ & $\frac{1}{10 ! 3 !}$ & $\frac{1}{10 ! 4 !}$ & $\frac{1}{10 ! 5 !}$ & $\frac{1}{10 ! 6 !}$ & $\frac{1}{10 ! 7 !}$ & $\frac{1}{10 ! 8 !}$ \\
\hline
\end{tabular}

Table 5: Coefficient Table of $\mathrm{U}(\mathrm{k}, \mathrm{h})$

$$
\begin{array}{r}
-\sum_{r=0}^{k} \sum_{s=0}^{h}(k-r+1) U(r, h-s) U(k-r+1, s), \\
U(0,0)=1, \quad U(k, 0)=\frac{(-1)^{k}}{k !}, \quad k \geq 1 .
\end{array}
$$

Using Table 4 and equation (2) we receive the closed form of solution:

$$
u(x, t)=e^{t-x}
$$



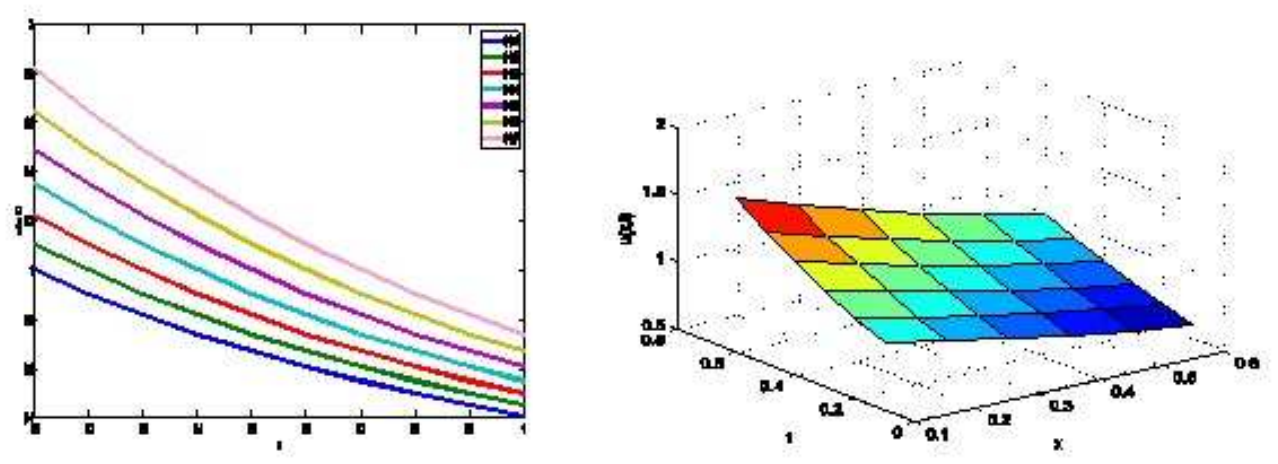

Figure 4: Graphical representation of $u(x, t)$ w.r.t time and distance

\section{Conclusion}

In this paper Differential Transform Method is successfully applied for solving Convection Diffusion and non- linear Reaction Diffusion convection equation. The advantage of DTM over other existing methods is that it is applied without using linearization, discretization or there no need to calculate the Adomain polynomial. DTM converts the linear or non-linear partial differential equation recursive formula. Thus result show that DTM is a powerful tool for solving linear and non-linear partial differential equation.

\section{References}

[1] F. Ayaz, On the two-dimensional differential transform method, Applied Mathematics and Computation, 23 (2003), 361-374, doi: : 10.1016/S0096-3003(02)00368-5.

[2] M.-J. Jang, C.-L. Chen, and Y.-C. Liu., Two-dimensional differential transform for partial differential equations, Applied Mathematics and Computation, 23 (2001), 261-270, doi: : 10.1016/S0096-3003(99)00293-3.

[3] C.K. Chen and S. H. Ho,Solving partial differential equations by two dimensional differential transform method, Applied Mathematics and Computation no. 2, 20 (2009), 131-164, doi: 10.1016/S0096-3003(98)10115-7.

[4] Mehdi Tatari, Mehdi Dehghan and Mohsen Razzaghi, Application of the Adomian decomposition method for the FokkerPlanck equation, Mathematical and Computer Modelling, 45 (2007), 639-650, doi: 10.1016/j.mcm.2006.07.010.

[5] Gupta, Sumit, Devendra Kumar, and Jagdev Singh, Analytical solutions of convectiondiffusion problems by combining Laplace transform method and homotopy 
perturbation method, Alexandria Engineering Journal, 54 (2015), 645-651, doi: 10.1016/j.aej.2015.05.004.

[6] Mohamed-Jawad, Anwar Ja'afar, Cenap Ozel, and Adem Kilicman, Variational iteration method in solving evolution equations, In: Aip Conference Proceedings (2010), doi: $10.1063 / 1.3525153$.

[7] Daga Amruta and V. H. Pradhan, Variational homotopy perturbation method for solving nonlinear reaction-diffusion convection problems, International Journal of Advanced Engineering Research and Studies, bf 2 (2013), 11-14. 